\title{
SENSEI: First Direct-Detection Constraints on Sub-GeV Dark Matter from a Surface Run
}

\author{
Michael Crisler, ${ }^{1, *}$ Rouven Essig, ${ }^{2, \dagger}$ Juan Estrada, ${ }^{1, \ddagger}$ Guillermo Fernandez, ${ }^{1, \S}$ Javier Tiffenberg, ${ }^{1, \|}$ \\ Miguel Sofo Haro, ${ }^{1,3, \uparrow}$ Tomer Volansky, ${ }^{4,5 * *}$ and Tien-Tien $\mathrm{Yu}^{6,7, \dagger}$
}

(SENSEI Collaboration)

\author{
${ }^{1}$ Fermi National Accelerator Laboratory, Post Office Box 500, Batavia, Illinois 60510, USA \\ ${ }^{2}$ C.N. Yang Institute for Theoretical Physics, Stony Brook University, Stony Brook, New York 11794, USA \\ ${ }^{3}$ Centro Atómico Bariloche, CNEA/CONICET/IB, Bariloche S4140, Argentina \\ ${ }^{4}$ Raymond and Beverly Sackler School of Physics and Astronomy, Tel-Aviv University, Tel-Aviv 69978, Israel \\ ${ }^{5}$ School of Natural Sciences, The Institute for Advanced Study, Princeton, New Jersey 08540, USA \\ ${ }^{6}$ Theoretical Physics Department, CERN, CH-1211 Geneva 23, Switzerland \\ ${ }^{7}$ Department of Physics and Institute of Theoretical Science, University of Oregon, Eugene, Oregon 97403, USA
}

(Received 8 April 2018; revised manuscript received 2 July 2018; published 8 August 2018)

\begin{abstract}
The Sub-Electron-Noise Skipper CCD Experimental Instrument (SENSEI) uses the recently developed Skipper-CCD technology to search for electron recoils from the interaction of sub-GeV dark matter particles with electrons in silicon. We report first results from a prototype SENSEI detector, which collected $0.019 \mathrm{~g}$ day of commissioning data above ground at Fermi National Accelerator Laboratory. These commissioning data are sufficient to set new direct-detection constraints for dark matter particles with masses between $\sim 500 \mathrm{keV}$ and $4 \mathrm{MeV}$. Moreover, since these data were taken on the surface, they disfavor previously allowed strongly interacting dark matter particles with masses between $\sim 500 \mathrm{keV}$ and a few hundred $\mathrm{MeV}$. We discuss the implications of these data for several dark matter candidates, including one model proposed to explain the anomalously large $21-\mathrm{cm}$ signal observed by the EDGES Collaboration. SENSEI is the first experiment dedicated to the search for electron recoils from dark matter, and these results demonstrate the power of the Skipper-CCD technology for dark matter searches.
\end{abstract}

DOI: 10.1103/PhysRevLett.121.061803

Introduction.-Identifying the nature of dark matter (DM) is one of the most important tasks of particle physics today, and direct-detection experiments play an essential role in this endeavor. The search for DM particles with masses $\lesssim 1 \mathrm{GeV}$ represents a new experimental frontier [1]. Traditional direct-detection searches, which are sensitive to recoil energy generated from DM scattering off of nuclei, typically have very little sensitivity to sub-GeV mass DM. Indeed, the best current bounds are limited to very large cross sections below $1 \mathrm{GeV}$ and are absent below $\sim 120 \mathrm{MeV}$ [2-4]. As suggested in Ref. [5], improved sensitivity to DM masses well below the GeV scale is possible by searching for signals induced by inelastic processes, for which a DM particle is able to deposit much more energy compared to the elastic scattering off of nuclei.

Published by the American Physical Society under the terms of the Creative Commons Attribution 4.0 International license. Further distribution of this work must maintain attribution to the author(s) and the published article's title, journal citation, and DOI. Funded by SCOAP ${ }^{3}$.
In particular, one of the most promising avenues is to search for one or a few ionized electrons that are released due to DM particles interacting with electrons in the detector.

Background-free searches for single or few-electron events are experimentally challenging. Sensitivity to such events has been demonstrated using two-phase time projection chambers with noble liquid targets, using data from XENON10 [6-8], XENON100 [7,9], and DarkSide-50 [10]. Significant progress can be made by utilizing solidstate detectors, which exhibit much lower thresholds due to their low, $\mathcal{O}(\mathrm{eV})$, band gaps. Recently, silicon chargecoupled devices (CCDs) with a special "Skipper" readout stage [11] and high-voltage cryogenic silicon detectors with transition edge sensor readout [12] have demonstrated single-electron sensitivity. The $\sim 1.1 \mathrm{eV}$ band gap of silicon allows for a DM mass threshold that is an order of magnitude lower than that achieved in noble liquid detectors, and permits significantly larger DM-electron scattering rates $[5,7,13-15]$.

The Sub-Electron-Noise Skipper CCD Experimental Instrument (SENSEI) is designed to utilize the SkipperCCD technology demonstrated in Ref. [11] to search for 
electron recoils from sub-GeV DM. While the ultimate goal of the SENSEI Collaboration is to build a 100-g detector consisting of multiple Skipper-CCDs, a prototype detector is currently operating $\sim 100 \mathrm{~m}$ underground near the MINOS experiment at Fermi National Accelerator Laboratory (Fermilab). This prototype was first tested on the surface, collecting 0.019-g day of data (before analysis cuts).

In this Letter, we present the first constraints on sub-GeV DM derived from SENSEI commissioning data. We exclude novel parameter space for DM masses below $\sim 4 \mathrm{MeV}$, above which the XENON10 constraint from Refs. [6,7] dominates. Furthermore, operating on the surface allows a search for DM that strongly interacts with the visible sector. Such DM does not penetrate the Earth, and detectors placed deep underground, such as the nobleliquid detectors mentioned above, have no sensitivity. Despite large cosmic-ray backgrounds, this region can be easily probed by a detector on the surface with a small amount of data. The SENSEI data thus also place novel constraints on DM particles with masses of several hundred $\mathrm{MeV}$.

The SENSEI prototype detector.-We use a single Skipper-CCD of active area $1.086 \mathrm{~cm} \times 1.872 \mathrm{~cm}$ with an initial active mass of $0.0947 \mathrm{~g}$ of silicon fabricated parasitically in a production run for astronomical CCDs. The Skipper-CCD was packaged in a light-tight copper housing that was cooled to an estimated $130 \mathrm{~K}$ to reduce the dark current on the sensor and to reduce the emission of infrared photons from blackbody radiation [16]. The sensor was read by a modified Monsoon electronics system described in Ref. [11].

We analyze here a small amount of commissioning data, taken on May 11, 2017, at the Silicon Detector Facility (SiDet) at Fermilab. SiDet has an elevation of $\sim 220 \mathrm{~m}$ above sea level and a roof consisting of about $7.6 \mathrm{~cm}$ of concrete, $2 \mathrm{~mm}$ of aluminum, and $1 \mathrm{~cm}$ of wood. The thickness of the light-tight copper housing in which the sensor was placed is $3 \mathrm{~mm}$.

The Skipper-CCD is divided into four equal-size quadrants, read continuously, independently, and in parallel for $427 \mathrm{~min}$. One of the quadrants had unusually high singlesample readout noise due to a charge transfer inefficiency problem in the readout stage, and all data from it were immediately discarded, leaving an active mass of $0.071 \mathrm{~g}$. The other three had a single-sample readout noise of $\sim 4 e^{-}$; taking 800 samples reduced the noise to $\sim 0.14 e^{-}$. It took $t_{\text {pix }} \simeq 19.5495 \times 10^{-3} \mathrm{~s}$ to take 800 samples of a single pixel.

Each quadrant consists of 624 rows of 362 pixels. Each pixel has an area of $15 \mu \mathrm{m} \times 15 \mu \mathrm{m}$, a thickness of $200 \mu \mathrm{m}$, and a mass of $1.0476 \times 10^{-7} \mathrm{~g}$. The total data are saved in 18 "images" for each quadrant consisting of 200 rows of 362 pixels. Before data taking, the CCD is cleaned, removing any excess charge on all pixels. The first 624 recorded rows in each quadrant then have pixels whose exposure grows linearly from $t_{\text {pix }}$ to $624 \times 362 \times t_{\text {pix }}=73.6 \mathrm{~min}$, with the pixels in the following $(18 \times 200-624=2976)$ rows having a uniform exposure of $73.6 \mathrm{~min}$ each. Note that due to the continuous readout, each row subsequent to the first 624 rows has a uniform exposure, corresponding to the time it takes to move a charge packet across the entire array of the CCD quadrant. The total exposure for the 3 "active" quadrants is $0.019 \mathrm{~g}$ day.

Data selection.-The prototype Skipper-CCD has a measured dark current of $\sim 1.14 e^{-} / \mathrm{pixel} / \mathrm{day}$. This leads to a large number of 1-, 2-, and 3-electron events. In this Letter, we place conservative limits by assuming that all events are from DM.

After data collection, we implemented several standard quality cuts for CCD-based detectors $[17,18]$ as well as cuts specific to our analysis, whose selection efficiencies are listed in Table I for electron bins 1-5: (1) Single-pixel events. To simplify our analysis, we select only pixels whose neighboring pixels are empty. (i) DM within a single pixel.-While a DM event consists of one or more electrons that are created initially in a single pixel, the electrons can drift apart as they diffuse to the surface, allowing some electrons to diffuse to a neighboring pixel. The lateral drift distance is described by a Gaussian distribution with a standard deviation proportional to the transit time from the interaction point to the surface of the CCD [19]. The diffusion parameter can be measured directly from tracks produced by atmospheric muons [18]. For our CCD, the diffusion parameter is proportional to $0.002 / \mu \mathrm{m}$ times the interaction depth. The probability for the DM event to be contained in a single pixel is unity for 1-electron events, and drops to 0.166 for 100 -electron events. (ii) Nearest neighbor.-We remove all pixels that are next to an occupied pixel in the data; this cut also removes all tracks and clusters. (2) Noise. We veto images in which the readout noise is $30 \%$ larger than the expected readout noise as inferred from an over-scan region in which virtual (nonexistent) pixels are read. (3) Bleeding. At low temperatures the electron mobility may be impeded, implying a

TABLE I. Efficiencies for the data selection cuts for events with 1 to 5 electrons. The bottom row lists the number of observed events after cuts.

\begin{tabular}{llllll}
\hline \hline & \multicolumn{5}{c}{$N_{\mathrm{e}, \min }$} \\
\cline { 2 - 6 } Cuts & 1 & 2 & 3 & 4 & 5 \\
\hline 1. DM within a single pixel & 1 & 0.62 & 0.48 & 0.41 & 0.37 \\
2. Nearest Neighbor & 0.8 & 0.8 & 0.8 & 0.8 & 0.8 \\
3. Noise & 0.88 & 0.88 & 0.88 & 0.88 & 0.88 \\
4. Bleeding & 0.95 & 0.95 & 0.95 & 0.95 & 0.95 \\
Total & 0.67 & 0.41 & 0.32 & 0.27 & 0.24 \\
Number of events & 140302 & 4676 & 131 & 1 & 0 \\
\hline \hline
\end{tabular}




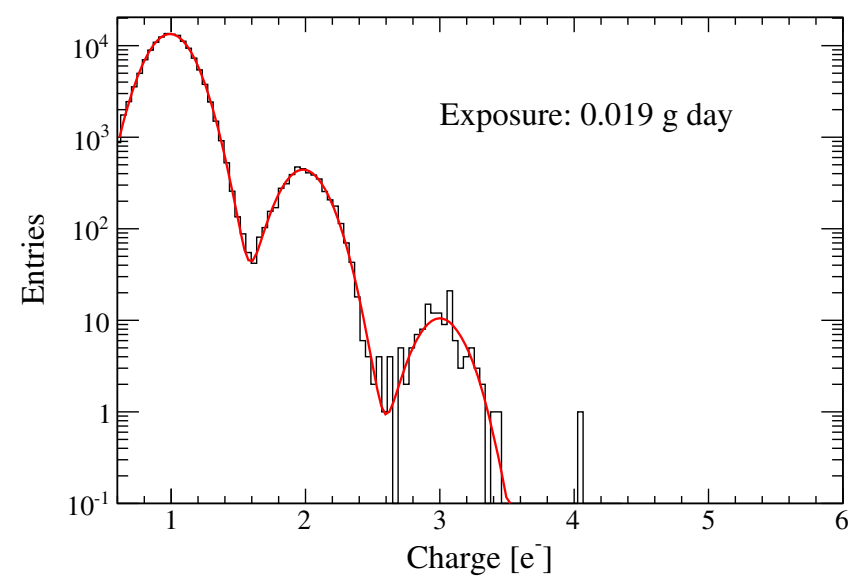

FIG. 1. Recorded spectrum after selection cuts for the $0.019 \mathrm{~g}$ day of commissioning data. Gaussian fits to the peaks show there are 140,302, 4676, 131, and 1 event(s) with 1, 2, 3, and 4 electrons, respectively. No events are seen for 5-100 electrons. The Gaussian width of the peaks are $\sim 0.14 e^{-}$.

small probability that an electron can get stuck in a given pixel for several downward shifts. If an event, such as a cosmic ray, produces a large number of electrons in some pixel(s), then pixels with several electrons may be found upstream in the image. We mask 10 pixels upstream of any pixel containing more than 100 electrons.

In what follows we bin the data, after the above selection cuts, according to the number of electrons per pixel, and derive constraints for each bin separately. The spectrum after cuts is shown in Fig. 1, together with Gaussian fits to the first three bins. We use the bins with 1-100 electrons in our analysis.
Analysis and results.-We calculate the DM recoil spectrum for several models, deriving constraints both on DM-electron scattering and on bosonic DM being absorbed by an electron [20-23]. For the scattering case, we use the calculations and conventions from [5,13], assuming a local DM density $\rho_{\mathrm{DM}}=0.4 \mathrm{GeV} / \mathrm{cm}^{3}$ [24]. We present our results in the $\bar{\sigma}_{e}$ versus $m_{\chi}$ parameter space for various DM form-factors, $F_{\mathrm{DM}}(q)$, where $m_{\chi}$ is the DM mass and $\bar{\sigma}_{e}$ is the cross section for DM to scatter off a free electron with the momentum transfer fixed to its typical value, $q=\alpha m_{e}$, where $\alpha$ is the fine-structure constant and $m_{e}$ is the electron mass. $F_{\mathrm{DM}}(q)$ parametrizes the model-dependent momentum dependence of the DM interaction: a "heavy" mediator with mass $\gg \alpha m_{e}$ has $F_{\mathrm{DM}}(q)=1$; an "ultralight" mediator with mass $\ll \alpha m_{e}$ has $F_{\mathrm{DM}}(q)=\left(\alpha m_{e} / q\right)^{2}$; and an electric-dipole-moment interaction with the standard model photon produces $F_{\mathrm{DM}}(q) \simeq \alpha m_{e} / q$.

For bosonic DM, we will consider that the DM is a dark photon, denoted $A^{\prime}$, with mass $m_{A^{\prime}}$, that is stable on the lifetime of the Universe. We follow the calculations and conventions in Ref. [21], and present results in the $\epsilon$ versus $m_{A^{\prime}}$ parameter space, where $\epsilon$ is the parameter that characterizes the strength of the kinetic mixing between the $A^{\prime}$ and the photon.

For each model, we calculate conservative $95 \%$ confidence level upper limits using Poisson statistics and assuming that all observed electrons in a given bin are DM events. We compare the resulting limit from each bin with the predicted number of DM events (for a given value of $\bar{\sigma}_{e}$ or $\epsilon$ ), after correcting for the efficiencies.

Our main results, for $\bar{\sigma}_{e}$ versus $m_{\chi}$ are shown in Fig. 2 for the three form factors discussed above. Despite a small
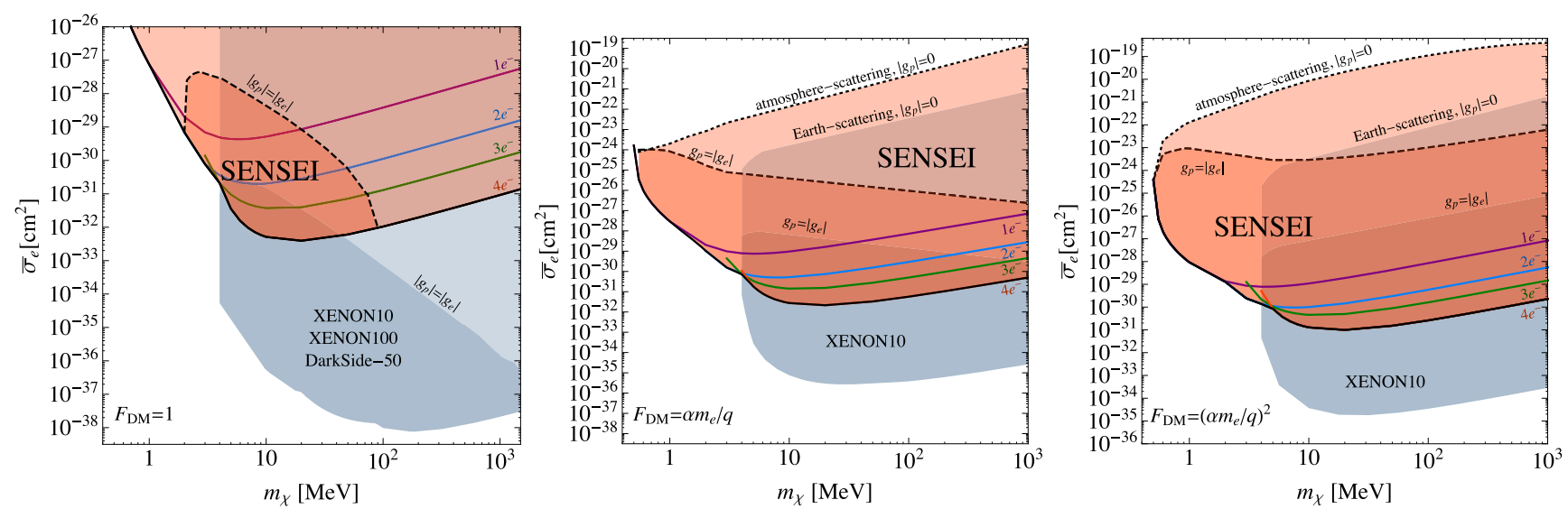

FIG. 2. The $90 \%$ C.L. constraints on the DM-electron scattering cross sections $\bar{\sigma}_{e}$ as a function of DM mass $m_{\chi}$ from a commissioning run above ground at Fermilab using the SENSEI prototype detector. We show different DM form factors, $F_{\mathrm{DM}}(q)=1, \alpha m_{e} / q$, and $\left(\alpha m_{e} / q\right)^{2}$. The purple, blue, green, and red lines correspond to the constraints from the 1-, 2-, 3-, or 4-electron bin, respectively. The black line is the minimum of these. The blue shaded regions are the current constraints from XENON10, XENON100, and DarkSide-50. For large cross sections, the DM is stopped in Earth's crust (atmosphere) and does not reach the noble-liquid (SENSEI prototype) detectors: the dark-shaded regions (labeled $\left|g_{p}\right|=\left|g_{e}\right|$ ) show order-of-magnitude estimates of the excluded parameter regions assuming the interaction between DM and ordinary matter is mediated by a heavy dark photon (left), an electric dipole moment (middle), or an ultralight dark photon (right). The light-shaded regions (labeled $g_{p}=0$ ) are order-of-magnitude estimates of the $90 \%$ C.L. excluded parameter regions assuming a mediator that couples only to electrons. 
exposure time on the surface, the SENSEI commissioning run already probes novel parameter space for light DM (mass $\lesssim 4 \mathrm{MeV}$ ) and for DM with large cross sections. This is the first time that a direct-detection constraint is derived for DM masses as low as $\sim 500 \mathrm{keV}$. In contrast, nobleliquid experiments (especially XENON10) probe lower cross sections for masses $\gtrsim 4 \mathrm{MeV}$.

In addition to having never probed DM masses below $4 \mathrm{MeV}$, the noble-liquid detectors that have previously constrained sub-GeV DM are operated underneath the Gran Sasso mountain. DM that interacts strongly with ordinary matter cannot reach these detectors due to scattering in the Earth. In contrast, much larger interaction strengths can be probed with the SENSEI surface run, as only the atmosphere (and a thin roof) can stop the DM.

The terrestrial effects on MeV-to-GeV DM scattering off nuclei or electrons are model dependent and have so far only been explored partially in the literature [25-27] (see Refs. [28-33] for larger DM masses). Here, we estimate the terrestrial effects at the order-of-magnitude level. A darkphoton mediator or electric-dipole-moment allows DM to scatter off nuclei and electrons in the atmosphere or Earth (we include elastic scatters only, ignoring inelastic scatters off electrons). In the darker shaded regions in Fig. 2 (labeled $\left|g_{p}\right|=\left|g_{e}\right|$ ) the respective detectors have no sensitivity. If the mediator only couples to electrons (and not to nuclei), a naive estimate leads to the excluded regions labeled " $g_{p}=0$ " (lighter shaded regions). We see that the SENSEI prototype constraints are largely complementary to existing noble-liquid detector constraints.

We give one example of a concrete model that can give rise to large cross sections in Fig. 3. We assume that a subdominant DM component, $\chi$, interacts with an ultralight dark photon $\left(m_{A^{\prime}} \ll \mathrm{keV}\right)$, with $\Omega_{\chi}=0.01 \Omega_{\mathrm{DM}}$. This model is motivated by the EDGES measurement of the $21-\mathrm{cm}$ spectrum at $z \simeq 17$, which revealed an anomalously large absorption signal $[34,35]$ (see also Refs. [36-51]). The SENSEI constraint (orange) is bounded by the solid (dashed) line for small (large) $\bar{\sigma}_{e}$. It disfavors novel parameter space for DM masses above a few hundred $\mathrm{MeV}$ for $\bar{\sigma}_{e} \gtrsim 10^{-25} \mathrm{~cm}^{2}$. Other constraints arise from the SLAC millicharge experiment [52], red-giant (RG), and horizontal-branch (HB) stars [53], the BBN and $\mathrm{CMB}$ measurements of the number of relativistic degrees of freedom $\left(N_{\text {eff }}\right)$ [53], and Supernova 1987A (SN) [54]. A more careful analysis of other possible constraints in this region is, however, warranted, including a re-analysis of low-threshold DM-nuclear recoil data [2,3], and an analysis of whether such a DM candidate would be evacuated from the Galactic disk by Galactic magnetic fields and supernova shock waves (as may be the case if the DM has a millicharge [55]).

We next present the SENSEI prototype constraints on $\epsilon$ versus $m_{A^{\prime}}$ for dark-photon $\mathrm{DM}\left(A^{\prime}\right)$, which can be absorbed by an electron, in Fig. 4. At small $\epsilon$, the SENSEI prototype

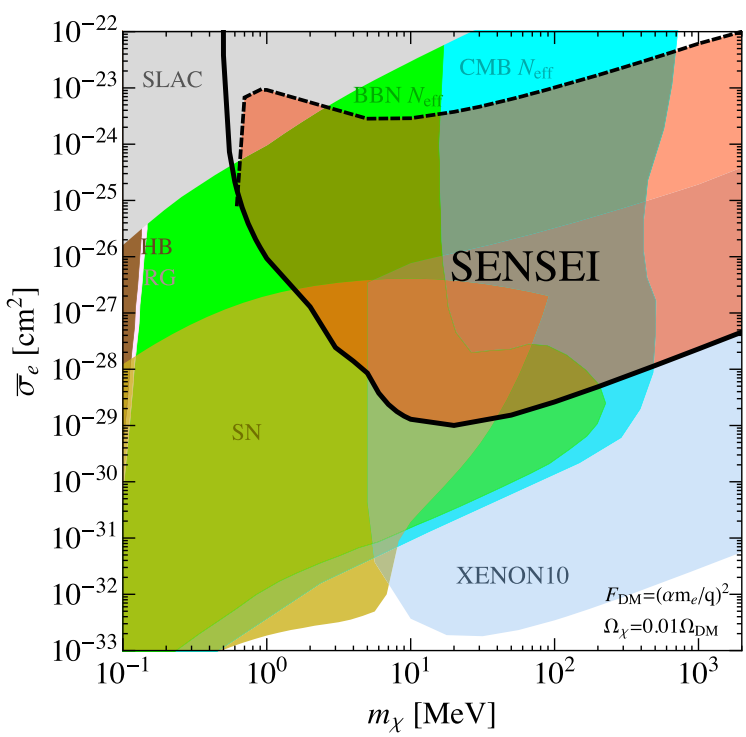

FIG. 3. The $90 \%$ C.L. constraint on $\bar{\sigma}_{e}$ versus $m_{\chi}$ for $F_{\mathrm{DM}}\left(q^{2}\right)=\left(\alpha m_{e} / q\right)^{2}$ from a surface run at Fermilab using the SENSEI prototype detector (orange region, bounded below by a solid line and above by a dashed line that is the same as the $\left|g_{p}\right|=\left|g_{e}\right|$ line in the right plot of Fig. 2). We assume that $\chi$ couples to an ultralight dark-photon mediator, and $\Omega_{\chi}=$ $0.01 \Omega_{\mathrm{DM}}$, which may explain the $21-\mathrm{cm}$ signal observed by EDGES. Other $90 \%$ C.L. constraints are described in the text. The SENSEI surface run disfavors a small region of previously open parameter space for $\bar{\sigma}_{e} \gtrsim 10^{-25} \mathrm{~cm}^{2}$ and $m_{\chi}$ greater than a few hundred $\mathrm{MeV}$.

constraint is weaker than other constraints due to its small exposure; however, for large $\epsilon$ new grounds are explored. We estimate the maximal coupling, $\epsilon_{\max }=1 / \sqrt{\rho L \sigma_{\mathrm{abs}}}$ above which the $A^{\prime}$ is absorbed by molecules in the atmosphere or by atomic electrons in Earth's crust and sensitivity is lost. Here $\sigma_{\mathrm{abs}}$ is the measured photoabsorption cross sections per molecule, $N$ is the average density, and $L$ is the depth. For simplicity, we take Earth's crust to consist of silicon with $\rho=2.7 \mathrm{~g} / \mathrm{cm}^{3}$, with $L=0.7$ (CDMSlite), 1.4 (XENON10/100), or $2 \mathrm{~km}$ (DAMIC). We take the atmosphere to consist of $\mathrm{O}_{2}$ and $\mathrm{N}_{2}$ [56] (with $\rho=1.2 \times 10^{-4} \mathrm{~g} / \mathrm{cm}^{3}$ and $L=86 \mathrm{~km}$ ). These dominate the absorption for $m_{A^{\prime}} \gtrsim 5 \mathrm{eV}$, the bond-dissociation energy of $\mathrm{O}_{2}$ (no data on $\sigma_{\mathrm{abs}}$ are available below $m_{A^{\prime}} \simeq 10 \mathrm{eV}$, so we extrapolate the available data down to $5 \mathrm{eV}$ ). For $m_{A^{\prime}} \lesssim 5 \mathrm{eV}$, ozone dominates the absorption, but its abundance is very small and does not affect the region shown in Fig. 4. We see that SENSEI closes a gap in laboratory probes of the large- $\epsilon$ region: the gap was bounded above by a measurement of the Rydberg constant (we show a $2 \sigma$ constraint adapted from Refs. [57-59]) and below by our analysis of terrestrial absorption effects for XENON10, XENON100, CDMSlite, and DAMIC [4,20-22,60] (stellar constraints [61,62] already disfavor this region). 


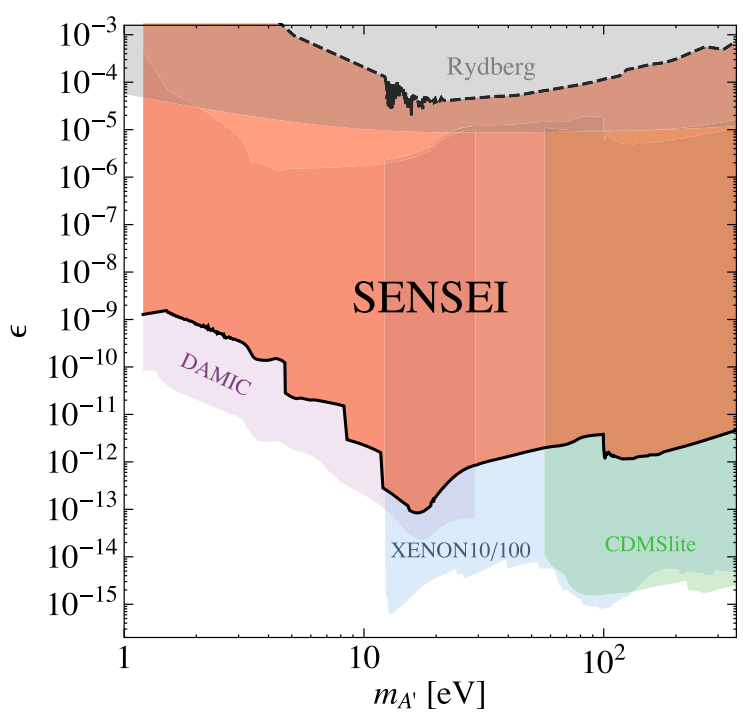

FIG. 4. $90 \%$ C.L. constraints on dark-photon DM absorption by electrons, in the kinetic mixing $(\epsilon)$ versus dark-photon mass $\left(m_{A^{\prime}}\right)$ parameter space from a surface run at Fermilab using the SENSEI prototype detector (orange). 90\% C.L onstraints from XENON10, XENON100, CDMSlite, DAMIC, and a measurement of the Rydberg constant are also shown. The direct-detection constraints are absent at large $\epsilon$, where the $A^{\prime}$ is absorbed in Earth's crust or atmosphere before reaching the detector. The SENSEI constraint closes a gap in laboratory probes of the high- $\epsilon$ region. We assume the $A^{\prime}$ to be DM, irrespective of any (modeldependent) production process.

Summary and outlook.-We present results from a lowexposure commissioning run of a SENSEI prototype detector. We demonstrate the first use of Skipper-CCD technology for a DM search, the first direct-detection constraints for DM masses $0.5-4 \mathrm{MeV}$, and the first direct-detection constraints on strongly interacting DM for masses between 0.5 and a few hundred MeV. Over the next few years, the SENSEI Collaboration aims to construct a detector consisting of $\sim 100 \mathrm{~g}$ of Skipper CCDs that are fabricated in a dedicated production run using highresistivity silicon. These detectors are expected to have a dark current several orders of magnitude lower than the prototype detector and an improved single-sample noise. We expect to collect an exposure that is almost $2 \times 10^{7}$ times larger than the exposure of the surface run and with far fewer background events, allowing us to explore vast new regions of DM parameter space.

We are grateful to Timon Emken, Chris Kouvaris, and Mukul Sholapurkar for extensive discussions and for letting us include preliminary results from an upcoming paper by RE, Emken, Kouvaris, and Sholapurkar. We also thank Alex Drlica-Wagner and Erez Etzion for carefully reading a draft of the paper and for numerous useful comments. We are grateful for the support of the Heising-Simons Foundation under Grant No. 79921. R. E. also acknowledges support from DOE Grant No. DE-SC0017938. This work was supported by Fermilab under DOE Contract No. DE-AC02-07CH11359. The work of T. V. is supported by the I-CORE Program of the Planning Budgeting Committee and the Israel Science Foundation (Grant No. 1937/12), by the European Research Council (ERC) under the EU Horizon 2020 Programme (ERC-CoG-2015Proposal No. 682676 LDMThExp), by the Israel Science Foundation-NSFC (Grant No. 2522/17) and by the German-Israeli Foundation (Grant No. I-1283-303.7/ 2014). T. V. is further supported by a grant from The Ambrose Monell Foundation, given by the Institute for Advanced Study. R. E. and T. V. are also funded by the Binational Science Foundation (Grant No. 2016153).

Note added.-Recently, Ref. [63] appears, showing constraints on DM-electron scattering that were obtained with a different technology and with different background challenges.

*mike@fnal.gov

†rouven.essig@stonybrook.edu

†estrada@fnal.gov

§fmoroni.guillermo@gmail.com

"javiert@fnal.gov

"miguelsofoharo@gmail.com

tomerv@post.tau.ac.il

†tien-tien.yu@cern.ch

[1] M. Battaglieri et al., arXiv:1707.04591.

[2] G. Angloher et al. (CRESST Collaboration), Eur. Phys. J. C 77, 637 (2017).

[3] F. Petricca et al. (CRESST Collaboration), Proceedings of the 15th International Conference on Topics in Astroparticle and Underground Physics (TAUP 2017) Sudbury, Ontario, Canada, 2017, arXiv:1711.07692.

[4] R. Agnese et al. (SuperCDMS Collaboration), Phys. Rev. Lett. 116, 071301 (2016).

[5] R. Essig, J. Mardon, and T. Volansky, Phys. Rev. D 85, 076007 (2012).

[6] R. Essig, A. Manalaysay, J. Mardon, P. Sorensen, and T. Volansky, Phys. Rev. Lett. 109, 021301 (2012).

[7] R. Essig, T. Volansky, and T.-T. Yu, Phys. Rev. D 96, 043017 (2017).

[8] J. Angle et al. (XENON10 Collaboration), Phys. Rev. Lett. 107, 051301 (2011); 110, 249901E (2013).

[9] E. Aprile et al. (XENON100 Collaboration), Phys. Rev. D 94, 092001 (2016); 95, 059901E (2017).

[10] P. Agnes et al. (DarkSide Collaboration), arXiv:1802 .06998 .

[11] J. Tiffenberg, M. Sofo-Haro, A. Drlica-Wagner, R. Essig, Y. Guardincerri, S. Holland, T. Volansky, and T.-T. Yu, Phys. Rev. Lett. 119, 131802 (2017).

[12] R. K. Romani et al., Appl. Phys. Lett. 112, 043501 (2018).

[13] R. Essig, M. Fernandez-Serra, J. Mardon, A. Soto, T. Volansky, and T.-T. Yu, J. High Energy Phys. 05 (2016) 046.

[14] S. K. Lee, M. Lisanti, S. Mishra-Sharma, and B. R. Safdi, Phys. Rev. D 92, 083517 (2015). 
[15] P. W. Graham, D. E. Kaplan, S. Rajendran, and M. T. Walters, Phys. Dark Universe 1, 32 (2012).

[16] The CCD temperature is measured with an RTD placed some distance away, resulting in an uncertainty of a few degrees.

[17] A. Aguilar-Arevalo et al. (CONNIE Collaboration), J. Phys. Conf. Ser. 761, 012057 (2016).

[18] A. Aguilar-Arevalo et al. (DAMIC Collaboration), Phys. Rev. D 94, 082006 (2016).

[19] S. E. Holland, D. E. Groom, N. P. Palaio, R. J. Stover, and M. Wei, IEEE Trans. Electron Devices 50, 225 (2003).

[20] H. An, M. Pospelov, J. Pradler, and A. Ritz, Phys. Lett. B 747, 331 (2015).

[21] I. M. Bloch, R. Essig, K. Tobioka, T. Volansky, and T.-T. Yu, J. High Energy Phys. 06 (2017) 087.

[22] Y. Hochberg, T. Lin, and K. M. Zurek, Phys. Rev. D 95, 023013 (2017).

[23] Y. Hochberg, T. Lin, and K. M. Zurek, Phys. Rev. D 94, 015019 (2016).

[24] P. Salucci, F. Nesti, G. Gentile, and C. F. Martins, Astron. Astrophys. 523, A83 (2010).

[25] T. Emken, C. Kouvaris, and I. M. Shoemaker, Phys. Rev. D 96, 015018 (2017).

[26] T. Emken and C. Kouvaris, J. Cosmol. Astropart. Phys. 10 (2017) 031.

[27] B. J. Kavanagh, R. Catena, and C. Kouvaris, J. Cosmol. Astropart. Phys. 01 (2017) 012.

[28] G. D. Mack, J. F. Beacom, and G. Bertone, Phys. Rev. D 76, 043523 (2007).

[29] B. J. Kavanagh, Phys. Rev. D 97, 123013 (2018).

[30] J. H. Davis, Phys. Rev. Lett. 119, 211302 (2017).

[31] M. S. Mahdawi and G. R. Farrar, J. Cosmol. Astropart. Phys. 12 (2017) 004.

[32] M. S. Mahdawi and G. R. Farrar, arXiv:1712.01170.

[33] D. Hooper and S. D. McDermott, Phys. Rev. D 97, 115006 (2018).

[34] J. D. Bowman, A. E. E. Rogers, R. A. Monsalve, T. J. Mozdzen, and N. Mahesh, Nature (London) 555, 67 (2018).

[35] R. Barkana, N. J. Outmezguine, D. Redigolo, and T. Volansky, arXiv:1803.03091.

[36] H. Tashiro, K. Kadota, and J. Silk, Phys. Rev. D 90, 083522 (2014).

[37] J. B. Muńoz, E. D. Kovetz, and Y. Ali-Haïmoud, Phys. Rev. D 92, 083528 (2015).
[38] R. Barkana, Nature (London) 555, 71 (2018).

[39] J. B. Muńoz and A. Loeb, Nature (London) 557, 684 (2018).

[40] A. Berlin, D. Hooper, G. Krnjaic, and S. D. McDermott, Phys. Rev. Lett. 121, 011102 (2018).

[41] S. Fraser et al., arXiv:1803.03245.

[42] G. D’Amico, P. Panci, and A. Strumia, Phys. Rev. Lett. 121, 011103 (2018).

[43] Z. Kang, arXiv:1803.04928.

[44] Y. Yang, X. Huang, and L. Feng, arXiv:1803.05803.

[45] M. Pospelov, J. Pradler, J. T. Ruderman, and A. Urbano, Phys. Rev. Lett. 121, 031103 (2018).

[46] M. Safarzadeh, E. Scannapieco, and A. Babul, Astrophys. J. 859, L18 (2018).

[47] S. Clark, B. Dutta, Y. Gao, Y.-Z. Ma, and L. E. Strigari, arXiv:1803.09390 [Phys. Rev. D (to be published)].

[48] K. Cheung, J.-L. Kuo, K.-W. Ng, and Y.-L. S. Tsai, arXiv: 1803.09398.

[49] T. R. Slatyer and C.-L. Wu, Phys. Rev. D 98, 023013 (2018).

[50] H. Liu and T.R. Slatyer, Phys. Rev. D 98, 023501 (2018).

[51] A. Falkowski and K. Petraki, arXiv:1803.10096.

[52] A. A. Prinz et al., Phys. Rev. Lett. 81, 1175 (1998).

[53] H. Vogel and J. Redondo, J. Cosmol. Astropart. Phys. 02 (2014) 029.

[54] J. H. Chang, R. Essig, and S. D. McDermott, arXiv:1803 .00993 .

[55] S. D. McDermott, H.-B. Yu, and K. M. Zurek, Phys. Rev. D 83, 063509 (2011).

[56] J. Fennelly and D. Torr, At. Data Nucl. Data Tables 51, 321 (1992).

[57] V. Popov, Turk. J. Phys. 23, 943 (1999).

[58] S. G. Karshenboim, Phys. Rev. D 82, 073003 (2010).

[59] S. G. Karshenboim, Phys. Rev. Lett. 104, 220406 (2010).

[60] A. Aguilar-Arevalo et al. (DAMIC Collaboration), Phys. Rev. Lett. 118, 141803 (2017).

[61] H. An, M. Pospelov, and J. Pradler, Phys. Lett. B 725, 190 (2013).

[62] J. Redondo and G. Raffelt, J. Cosmol. Astropart. Phys. 08 (2013) 034.

[63] R. Agnese et al. (SuperCDMS Collaboration), Phys. Rev. Lett. 121, 051301 (2018). 